\title{
Theoretical approaches and experimental evidence for liquid-vapor phase transitions in nuclei
}

\author{
L. G. Moretto*, J. B. Elliott*, L. Phair*, G. J. Wozniak*, C. M. Mader ${ }^{\dagger}$ and A. \\ Chappars $^{\dagger}$
}

${ }^{*}$ Nuclear Science Division, Lawrence Berkeley National Laboratory,

University of California, Berkeley, California 94720

'Hope College, Holland, MI 49423

\begin{abstract}
The leptodermous approximation is applied to nuclear systems for $T>0$. The introduction of surface corrections leads to anomalous caloric curves and to negative heat capacities in the liquid-gas coexistence region. Clusterization in the vapor is described by associating surface energy to clusters according to Fisher's formula. The three-dimensional Ising model, a leptodermous system par excellence, does obey rigorously Fisher's scaling up to the critical point. Multifragmentation data from several experiments including the ISiS and EOS Collaborations, as well as compound nucleus fragment emission at much lower energy follow the same scaling, thus providing the strongest evidence yet of liquid-vapor coexistence.
\end{abstract}

\section{INTRODUCTION}

The phase diagram of nuclear matter is a frequently displayed picture in modern presentations on nuclear physics. It is meant to show the known and expected states of aggregation of hadronic matter and their thermodynamic boundaries. Typically, it portrays the liquid to vapor transition as well as the transition from the hadronic phase to the quark-gluon plasma. Unfortunately, up to now, this diagram has been based more on educated guesses and artistic license than on experimental fact. We believe that we have succeeded in removing some of this arbitrariness by characterizing the liquid-vapor coexistence curve from low temperatures up to the critical point. What follows is an outline of the ideas that have lead us to this characterization.

Nuclei are leptodermous, mesoscopic clusters. Their thin skin leads naturally to an expansion of their energy in powers of $A^{-1 / 3}$. The leading term, proportional to the number of nucleons $A$, is the bulk term; the second, proportional to $A^{2 / 3}$, is the surface term, the next, proportional to $A^{1 / 3}$, is the curvature term, etc. This is the basis of the liquid drop model, which by merely correcting the bulk terms with a surface term, manages to reproduce the binding energies of nuclei to within $1 \%$. There is ample reason to believe that a similar leptodermous treatment of nuclear systems at $T>0$ should lead to an equivalently good reproduction of nuclear thermodynamical properties.

In particular, the appearance of a vapor phase at $T>0$ opens two complementary perspectives for the characterization of phase coexistence: the liquid perspective and the vapor perspective. From the liquid perspective, one is lead to consider the transition between a liquid-like nuclear droplet and its equilibrium vapor, with the attendant caloric curve, as well as compound nuclear decay in terms of enthalpy of vaporization. From the vapor perspective one is lead to consider the properties of the vapor phase, especially the extent to which nucleons are aggregated into clusters, as indicators of incipient liquid condensation.

In the first part of this presentation we take the liquid perspective and derive analytically the caloric curve and the (negative) heat capacity for a drop undergoing an isobaric phase transition. In the second part we take the vapor perspective and show that the clusterization in the three-dimensional Ising model can be accounted for in terms of the leptodermous expansion. The rigorous obeyance of the mass and temperature scaling law (Fisher's scaling) by the Ising model is exactly paralleled by the nuclear systems in multifragmentation, thus providing the best evidence yet for liquid-vapor coexistence. Finally, we revert to the liquid perspective and show how compound nucleus fragment emission can be reconciled with the liquid to vapor phase transition. 


\section{THE ISOBARIC PHASE TRANSITION OF A LIQUID DROP}

The thermodynamical equilibrium properties of first order phase transitions are completely describable in terms of the thermodynamic state variables associated with the individual separate phases. For this reason, in contrast with continuous phase transitions, first order phase transitions are "trivial," and interesting only in so far as they herald the appearance of a hitherto unknown or undescribed phase.

Renewed attention to phase transitions has been generated by studies of models with well defined Hamiltonians with either short range interactions (e.g. the Ising model $[1,2,3,4,5,6,7,8,9,10,11,12]$ or the lattice gas model $[13,14,15,16,17,18,19,20,21,22,23,24,25])$ or incorporating long range interactions such as gravitation or electro-magnetic interactions. Features expected to disappear in the thermodynamic limit, if such a limit exists, were noticed and were claimed to be essential, characteristic indicators of phase transitions in mesoscopic systems [26]. For instance, first order phase transitions were associated with anomalous convex intruders in the entropy versus energy curves, resulting in back-bendings in the caloric curve, and in negative heat capacities [26].

These anomalies have been attributed to a variety of causes, the foremost of which are surface effects, and long range forces [26]. Unfortunately however, the numerical nature of the calculations tends to make the identification of the causes of negative heat capacities rather problematic.

In the context of nuclear physics, microcanonical models of nuclear multifragmentation have associated the anomalies of a convex intruder with the onset of multifragmentation [26]. Furthermore, lattice gas models in the isobaric regime have also shown negative specific heats in the coexistence region, where multifragmentation also appears [22]. The question of whether the two transitions are related and possibly coincident with the liquid-vapor transition is still very much open.

Recently, the claim has been made of an empirical observation of these anomalies, such as negative heat capacities in nuclear systems [27]. These negative heat capacities have been inferred from the study of fluctuations in multifragmenting nuclear systems. It would be highly desirable to ground any evidence for these anomalies, theoretical or otherwise, on thermodynamics itself, minimally modified to allow for the possible role of surface effects related to the finiteness of the system.

In this section we illustrate analytically how effects such as negative heat capacities can arise within a standard thermodynamic treatment. We consider the evaporation of a drop of ordinary liquid. Our only concern with mesoscopicity is the explicit treatment of the surface of the drop.

No qualitative difference in the picture results by considering a drop of finite radius $r$. The only difference is that the overall free energy of the drop as we shall see below, is higher than that of the bulk and the equilibrium vapor pressure is correspondingly higher.

The state of equilibrium between a liquid and its vapor can be described in the simplest way by the Clapeyron Equation

$$
\frac{d p}{d T}=\frac{\Delta H_{m}}{\Delta V_{m} T}:
$$

where, $p$ and $T$ are the pressure and temperature, $\Delta H_{m}$ is the molar enthalpy of vaporization and $\Delta V_{m}$ is the difference of the molar volumes of vapor, $V_{m}^{v}$, and liquid, $V_{m}^{l}$.

Specialization to the case of a drop of radius $r$ can be achieved by modifying the enthalpy to account for the surface energy [28]

$$
\Delta H_{m}=\Delta H_{m}^{0}-c_{s} S_{m}^{l}=\Delta H_{m}^{0}-\frac{3 c_{s} V_{m}^{l}}{r}
$$

where $\Delta H_{m}^{0}$ is the "bulk" molar enthalpy, $S_{m}^{l}$ and $V_{m}^{l}$ are the surface and volume of the drop and $c_{s}$ is the surface energy coefficient.

Neglecting $V_{m}^{l}$ compared to $V_{m}^{v}$ and considering the vapor ideal, i.e. $V_{m}^{v}=T / p$, we can integrate Eq. (1), assuming also $\Delta H_{m}$ to be constant. We obtain

$$
p=p_{0} \exp \left(-\frac{\Delta H_{m}^{0}}{T}+\frac{3 c_{s} V_{m}^{l}}{r T}\right)
$$

or

$$
p=p_{\text {bulk }} \exp \left(\frac{3 c_{s} V_{m}^{l}}{r T}\right) .
$$

This equation contains all the thermodynamical information necessary to characterize the phase coexistence of the liquid drop of radius $r$ with its vapor. The salient feature is the rise of the vapor pressure with decreasing radius. 

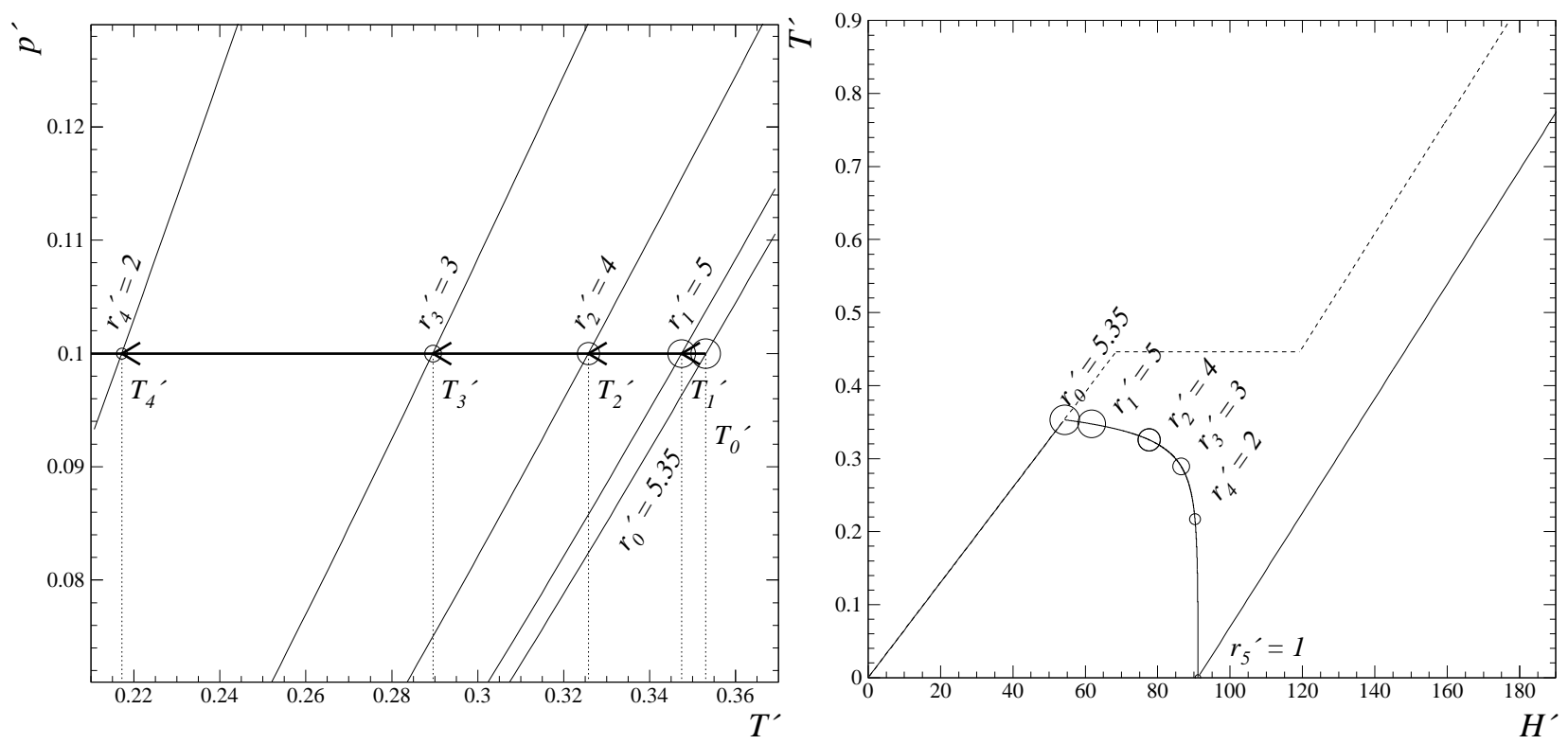

FIGURE 1. Left: Saturated vapor pressure as a function of the temperature for different droplet radii. The size of the open circles is proportional to the droplet radius. Right: The history dependent caloric curve of an evaporating drop at constant pressure. Dashed lines represent bulk behavior, solid line shows the drop's caloric curve. The scaled enthalpy is $H^{\prime}=H\left(\Delta H_{m}^{0} / 3 c_{s} V_{m}^{l}\right)^{3} /\left(4 \pi / V_{m}^{l}\right)$.

Fig. 1 gives a map of the function $p^{\prime}=p^{\prime}\left(T^{\prime}, r^{\prime}\right)$, in terms of the scaled variables

$$
p^{\prime}=\frac{p}{p_{0}}, T^{\prime}=\frac{T}{\Delta H_{m}^{0}}, r^{\prime}=\frac{\Delta H_{m}^{0}}{3 c_{s} V_{m}^{l}} r
$$

For any given radius $r$, the function $p=p(T, r)$ describes the equilibrium condition between the drop and its vapor. In other words, it is the phase diagram of the drop.

Let us now introduce some history and construct a caloric curve for a drop of radius $r_{0}$ at constant pressure $p_{0}$. As the drop is heated, and before the vapor can appear, the temperature increases according to $\Delta H=C_{p}^{l} \Delta T$, with $C_{p}^{l}$ is the liquid's heat capacity and is approximately constant. When $T$ reaches the value $T_{0}$ at which the vapor pressure $p\left(r_{0}\right)=p_{0}$, the vapor first appears and it expands against the container. The heat of vaporization is absorbed at a rate $H_{m}\left(r_{0}\right)$. However, as it evaporates, the drop sees its radius decreasing from its initial value, chosen to be $r_{0}^{\prime}=5.35$. At constant temperature the vapor pressure would rise, but, at constant pressure, as we are now operating, the temperature decreases as shown in Fig. 1, as the system absorbs its heat of vaporization, so that,

$$
\Delta H=\int_{r}^{r_{0}} \Delta H_{m} \frac{d V}{V_{m}^{l}}=\frac{4 \pi}{V_{m}^{l}}\left(\frac{3 c_{s} V_{m}^{l}}{\Delta H_{m}^{0}}\right)^{3}\left[\frac{1}{3}\left(r_{0}^{\prime 3}-r^{\prime 3}\right)-\frac{1}{2}\left(r_{0}^{\prime 2}-r^{\prime 2}\right)\right]
$$

and

$$
T^{\prime}=T_{0}^{\prime}\left(\frac{1-\frac{1}{r^{\prime}}}{1-\frac{1}{r_{0}^{\prime}}}\right)
$$

After the drop has totally evaporated, the vapor can increase its temperature according to $\Delta H=C_{p}^{v} \Delta T$ where $C_{p}^{v}$ is the vapor heat capacity at constant pressure. The resulting caloric curve defined parametrically by Eq. (6) and Eq. (7) and shown in Fig. 1 is rather interesting. It has a decreasing branch associated with the phase transition, along which the heat capacity is negative!

As an aside we note that the scaled radius $r^{\prime}$ is just the ratio of the bulk energy to the surface energy. Thus for a nuclear system the range shown: $1 \leq r_{0}^{\prime} \leq 5.35$, corresponds to a gold nucleus $(A=197)$ evaporating to a single nucleon.

These rather extraordinary features are wholly due to the interesting but, in a way, accidental history of the decreasing radius with increasing evaporation. 
Typically, experiments heat a preassigned system with a certain amount of energy $\Delta E$ or $\Delta H$, and determine the resulting change in entropy $S$ and temperature $1 / T=\partial S /\left.\partial E\right|_{V}$; from the resulting caloric curve, phase coexistence diagrams are extracted. However, as shown above, the evolution of the system occurring during heating introduces complications in the construction of a phase diagram from such a caloric curve.

To avoid this problem an experimentalist or theorist would have to keep the radius constant or correct for its change while determining the vapor pressure as a function of the temperature, thereby eliminating the accidental aspects associated with the evolution of the system.

The results obtained here are firmly grounded on thermodynamics with a straightforward accounting of finiteness through the surface correction. They are exact in the limit in which the liquid drop model holds, namely, down to nuclei/clusters containing 20 or so constituents. They are completely general, as they do not depend on specific details of the system but rather on its gross properties. In fact, they should be used as the paragon for lattice gas models and the like. In the limit in which these models represent liquid vapor coexistence, they must reproduce the present results.

Even more importantly, this approach obviates the need for repeating numerical calculations for each individual system or drop size. All that is required is to determine the bulk energy (enthalpy) and the surface energy coefficient of a given phase once and for all.

It is worth repeating that, once the constraint of constant pressure is enforced, the results described here are entirely general, as they apply to any small system undergoing solid-vapor or liquid vapor transitions.

\section{THE THREE-DIMENSIONAL ISING MODEL: A PARADIGM OF LIQUID-VAPOR COEXISTENCE IN NUCLEAR MULTIFRAGMENTATION}

Nuclear multifragmentation is a process occurring at the limits of nuclear excitation, and, as such, portrays an appropriate richness and complexity. While the fundamental problem of dynamics vs. statistics is still debated, it appears ever more clearly that many thermal/statistical features underlie the empirical body of data. In particular, two features associated with the fragment multiplicities are found to be quite pervasive in all multifragmentation reactions. They have been named "reducibility" and "thermal scaling" [28, 29, 30].

Reducibility is the property that the probability of observing $n$-fragments of a given size is expressible in terms of an elementary one-fragment probability. This property can occur only if fragments are created independently from one another and it coincides with stochasticity. Both binomial, and its limiting form, Poissonian reducibilities have been extensively documented experimentally for nuclear multifragmentation [28, 29, 30].

Thermal scaling is the linear dependence of the logarithm of the one-fragment probability with $1 / T$ (an Arrhenius plot). It indicates that the emission probability for fragment type $i$ has a Boltzmann dependence

$$
q_{i}=q_{0} e^{-B_{i} / T}
$$

where $B_{i}$ is a "barrier" corresponding to the production process.

The combination of these two empirical features powerfully attests to a statistical mechanism of multifragmentation in general, and to liquid-vapor coexistence specifically [31].

Many statistical models have been proposed as an explanation for multifragmentation. It is our intention to identify a model which is as simple as possible, and yet captures the essential features observed in the experiments. Percolation in its many versions has been widely used $[32,33,34,35]$. However, while being simple, it does not lend itself to a nontrivial thermal study [36]. The three-dimensional Ising model satisfies both the criteria of simplicity in its Hamiltonian and lends itself to a thermal treatment with nontrivial results.

While the Ising model has been widely studied in terms of its continuous phase transition $[1,2,3,4,5,6,7,8,9$, $10,11,12]$ and has even been used to study nuclear systems $[15,16,17,18,19,20,21,22,23,24,25]$, the problem of clusterization, of paramount interest to us here, has received relatively little attention. We will show that this model contains both the features of reducibility and thermal scaling observed in nuclear multifragmentation. In showing the features of thermal scaling we will demonstrate that for temperatures below the critical temperature, the slopes of the Arrhenius plots associated with the individual masses of the fragments, or the "barriers", portray a dependence on the fragment mass $(A)$ of the form $B \propto A^{\sigma}$, where $\sigma$ is a critical exponent which relates the mass to the cluster surface. In addition, the individual Arrhenius plots for each fragment mass can be absorbed into a single scaling function identical to that of Fisher's droplet model [37, 38, 39, 40, 41], which defines the liquid-vapor coexistence line up to the critical temperature. 
The Hamiltonian of the Ising model has two terms: the interaction between nearest neighbor (n.n.) spins in a fixed lattice and the interaction between the fixed spins and an external applied field $H_{\text {ext }}$ :

$$
H=-J \sum_{i, j=\{n . n .\}} s_{i} s_{j}-H_{e x t} \sum_{i} s_{i}
$$

where $J$ is the strength of the spin-spin interaction. In the absence of an external magnetic field, the system exhibits a first-order phase transition for temperatures up to the critical point at which it exhibits a continuous phase transition. The critical temperature for the three-dimensional Ising model has not been determined analytically; however, Monte Carlo techniques have yielded a value of $T_{c}=4.513 \mathrm{~J} / k_{b}$ [4].

The phase transition of the zero-field Ising model is equivalent to the Lattice gas model $[13,14]$. The positive spins are mapped to unoccupied sites in a lattice gas and the negative spins are mapped to occupied sites. The phase transition is then analogous to a liquid-vapor phase transition. If a correspondence is observed between features of the Ising model and nuclear multifragmentation, it would strengthen the case for nuclear multifragmentation being the signature for a liquid-gas phase transition of excited nuclei.

In the present study, Monte Carlo techniques are used to determine equilibrium cluster distributions in a canonical ensemble as a function of temperature for a simple cubic lattice. Since we are interested in studying liquid-vapor coexistence, all calculations are performed at zero external field $\left(H_{\text {ext }}=0\right)$. The lattice contains $50^{3}$ spins, and periodic boundary conditions are used to minimize finite size effects. The Swendsen-Wang algorithm [7] was used to determine the equilibrium spin configurations of the lattice for given temperatures, and physical clusters were identified using the Coniglio-Klein [3] prescription.

We now proceed to analyze the Monte Carlo results in the same way as has been done with nuclear multifragmentation data $[28,29,30]$. We shall consider first whether the multiplicity distributions for individual fragments manifest Poissonian reducibility. Fig. 2 shows the multiplicity distributions for a sample of fragment sizes and temperatures. The solid lines represent Poisson distributions calculated from the corresponding mean multiplicities. The distributions are indeed remarkably close to Poissonian not only for the cases shown, but for all masses and all temperatures below, at and above $T_{c}$. Therefore Poissonian reducibility is empirically verified.

This signifies that the probability of finding $m$ clusters of size $A$ depends only on the probability of finding one cluster of that size and is nearly independent of the probability of finding clusters of any other size. This feature is also observed in percolation models, Fisher's droplet model and nuclear fragmentation [31].

If the fragment distributions exhibit thermal scaling, the distributions must be of the form given in Eq. (8). Thus in an Arrhenius plot (a semi-log graph of the number of clusters of size $A\left(n_{A}\right)$ vs. $1 / T$ ), the distributions should be linear.

As shown in Fig. 2, this is indeed the case over a wide range of temperatures $\left(0<T<T_{c}\right)$ and fragment sizes. While we have shown distributions for clusters up to size $A=100$, the trend continues for larger clusters, but with poorer statistics. This linearity extends over more than four orders of magnitude. It rigorously confirms the form of Eq. (8) and signifies the independent thermal formation of fragments controlled by a size-dependent "barrier". This feature has been amply verified in nuclear multifragmentation [28, 31, 41]. By fitting the linear regions of the fragment distributions below the critical temperature, the "barriers" can be extracted. The "barriers" for each cluster size are shown in Fig. 3.

These "barriers" find their origin in the number of broken bonds associated with a cluster which should be proportional to the surface area of the cluster itself. Therefore they should be well described by a power-law:

$$
B(A)=c_{0} A^{\sigma} \text {. }
$$

with $\sigma \simeq 2 / 3$. The fit of the extracted "barriers" is $B=(12.77 \pm 0.04) J A^{(0.723 \pm 0.008)}$ and is remarkably good (see Fig. 3). The error bars are statistical. Estimates of the errors associated with different analysis procedures (described below) increase the errors in $\sigma$ and $c_{0} / J$ to \pm 0.026 and \pm 1.2 respectively. The remarkable closeness of $c_{0} / J$ to $4 \pi$ has not escaped us.

The value for $\sigma$ determined from the "barriers" is close to $2 / 3$, the value one would expect for spherical clusters of closely packed spherical objects [6]. This picture leads naturally to the interpretation of $c_{0}$ as a surface energy coefficient. Previous studies have determined $\sigma$ from scaling relations between critical exponents and obtained a value of $0.64[2,6,37,38,39,40]$, though recent studies indicate 0.73 is a better value [12]. The methods used in the previous studies do not utilize the cluster distributions at all to determine $\sigma$, so it is gratifying that our value is in close agreement. Duflot, et. al. used a cluster method to determine $\sigma$ for an Ising model [24] and obtained values that are consistent with those found in this study. Thus our technique is a new and accurate method to determine the critical exponent $\sigma$, never before explored and leads to a novel evaluation of the surface energy coefficient. 

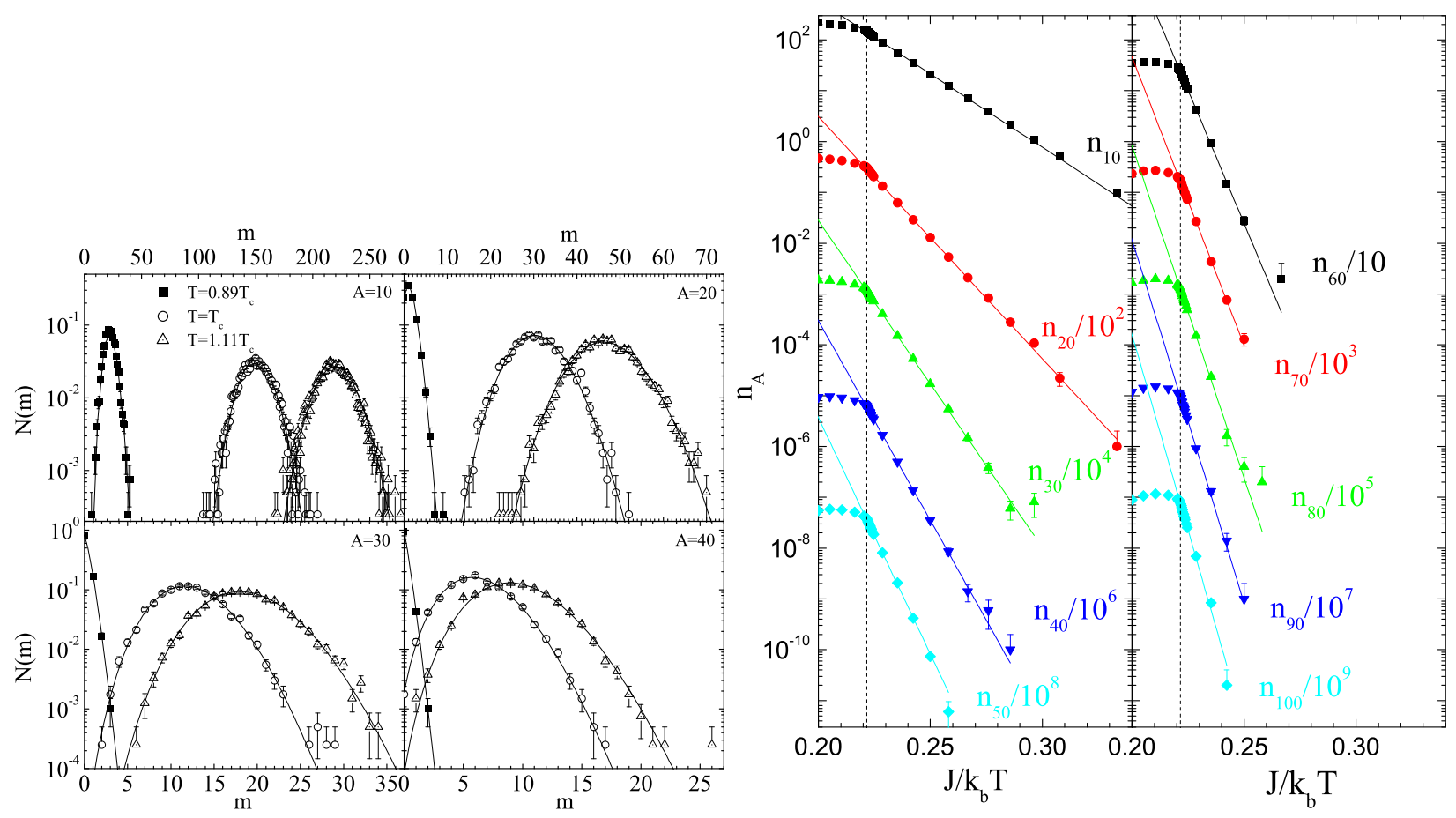

FIGURE 2. Left: The probability distributions for obtaining $m$ fragments of size $A$ at the three temperatures indicated. The solid lines are Poisson distributions with means given by the Monte Carlo data. Right: Arrhenius plots of the cluster distributions. A statistical error bar is shown when it exceeds the size of the data point. The lines are fits of the form given in Eq. (8). The critical temperature is indicated by the dashed line.
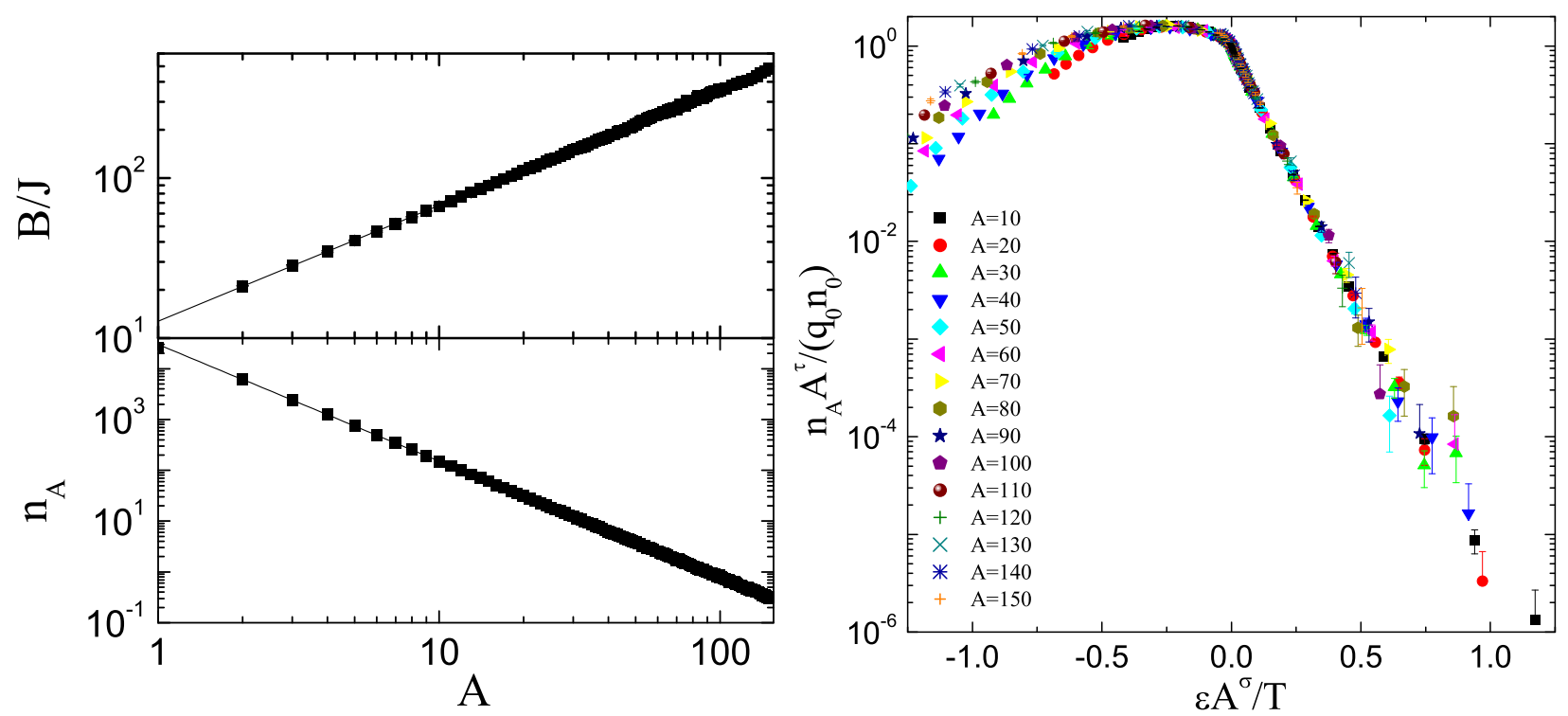

FIGURE 3. Left: The upper panel shows the extracted "barriers" from the fits to the cluster distributions. The line is a fit of the form given in Eq. (10). The lower panel shows the power law behavior of the cluster distribution at $k_{b} T / J=4.515$. The line is a fit of the form given in Eq. (12). In both panels, error bars are smaller than the data point. Right: Scaling behavior of cluster distributions. 
The features of reducibility and thermal scaling discussed above can be found united in Fisher's formula for the cluster abundance in a vapor as a function of cluster size and temperature. The formula is

$$
n_{A}(T)=q_{0} A^{-\tau} \exp \left(\frac{A \Delta \mu}{T}\right) \exp \left(\frac{c_{0} A^{\sigma}}{T_{c}}\right) \exp \left(-\frac{c_{0} A^{\sigma}}{T}\right)
$$

where $q_{0}$ is a normalization constant, $\tau$ is a topological critical exponent, $\Delta \mu$ is the difference in chemical potential of the system and the liquid and $c_{0}$ is the surface energy coefficient at zero temperature. The formula is valid for temperatures up to the critical point, at which point the surface energy of a cluster $\left(c_{0} A^{\sigma}\left(1-T / T_{c}\right)\right)$ vanishes. It contains the thermal scaling up to $T_{c}$ and the dependence of the "barrier" on the cluster mass through the critical exponent $\sigma[37,38,39,40]$. One can see that the cluster surface energy coefficient $\left(c_{0}\right)$ is also found directly in Fisher's droplet model. It is interesting to explore further the applicability of this formula to the Ising model.

In addition to the linear behavior of the Arrhenius plots below the critical temperature, the Fisher droplet model also predicts that the cluster size distribution at the critical point must follow a power law

$$
n_{A}\left(T_{c}\right)=q_{0} A^{-\tau}
$$

where $q_{0}$ is fixed by the normalization relationship

$$
q_{0}=\frac{\sum_{A=1}^{\infty} n_{A}\left(T_{c}\right)}{\sum_{A=1}^{\infty} A^{-\tau}} .
$$

Away from the critical temperature, the cluster distribution should not follow a pure power law. Thus, to determine $\tau$ without a prior knowledge of the critical temperature, linear fits to the cluster distributions were determined for all temperatures with $\tau$ and $q_{0}$ as free parameters. At the critical temperature, the fit should have the lowest $\chi^{2} / D o F$ and thus $\tau$ and $q_{0}$ are fixed by the fit. The best power law fit of the fragment abundances is shown in the lower panel of Fig. 3. The critical temperature was found to be $k_{b} T_{c} / J=4.515 \pm 0.011$ with a best fit of the form $n_{A}\left(T_{c}\right)=(30,000 \pm 5,000) A^{(-2.30 \pm 0.08)}$. This value of the critical temperature is consistent with the value determined for infinite systems $\left(4.513 \mathrm{~J} / k_{B}\right)$. The value of $\tau(2.30 \pm 0.08)$ is close to the expected value for an infinite system (2.21) and is consistent with the value found by Wang (2.27) for a larger lattice [6]. The value of $q_{0}=30,000$ can be compared to the prediction of Eq. (13) which yields 27, 566.

For the present calculations with $H_{\text {ext }}=0$ and an unconstrained magnetization, the system exists on the coexistence curve for $T<T_{c}$. Thus, the chemical potentials of the liquid and gas phases are equal ( $\left.\Delta \mu=0\right)$, and Eq. (11) can be rewritten as:

$$
n_{A}(T) A^{\tau} / q_{0}=\exp \left(-c_{0} A^{\sigma} \varepsilon / T\right)
$$

with $\varepsilon=\left(T_{c}-T\right) / T_{c}$. Therefore, a graph of the scaled cluster distributions $\left(n_{A}(T) A^{\tau} / q_{0}\right)$ as a function of $\varepsilon A^{\sigma} / T$ should collapse the distributions of all cluster sizes onto a single curve. This scaling behavior can clearly be seen in Fig. 3. This nearly perfect collapse below the critical temperature extends over six orders of magnitude for a broad range of cluster sizes and it is very linear. Thus the clustering in the three-dimensional Ising model can be described by Fisher's droplet model.

The Ising clusters constructed here can be properly thought of as "vapor" in equilibrium with the "liquid" percolating cluster. Coexistence of the two phases is determined by the observation that the empirical scaling implies $\Delta \mu=0$. The fact that both the three-dimensional Ising model and the experimental nuclear multifragmentation data obey the same scaling predicted by Fisher's droplet model indicates that nuclear multifragmentation can indeed be identified as the clustering (non-ideality) in a nuclear vapor in equilibrium with the nuclear liquid [31, 41].

The quality of the scaling shown in Fig. 3 led us to test our results by simultaneously fitting the cluster distributions in the Arrhenius plots using Fisher's model (Eq. 11) for temperatures up to the critical temperature. There are several methods for performing this fit. In one case, the values of $\tau$ and $T_{c}$ are fixed as determined in Fig. 3 while the values of $\sigma, c_{0}$ and $q_{0}$ are free. Another method allows all parameters $\left(\tau, \sigma, T_{c}, c_{0}\right.$ and $\left.q_{0}\right)$ to be determined in a single fit. The values obtained from both of these methods are consistent with those determined from the multistep method described earlier. Combining the results from all three methods allows us to estimate the errors associated with our technique. The resulting errors are those quoted previously in this work.

In conclusion, we have shown that the clusterization in the Ising model, like nuclear multifragmentation, portrays reducibility and thermal scaling. In addition, the Arrhenius plots allow for the extraction of "barriers" which are found to have a dependance of $B=c_{0} A^{\sigma}$, where $\sigma$ is a critical exponent. The "barrier" coefficient from the Arrhenius plots is equivalent to the surface energy coefficient of the clusters in Fisher's model. Thus we have found a new method for 
determining both the critical exponent $\sigma$ and the surface energy of the 3-D Ising model clusters directly from the cluster distributions. The reducibility and thermal scaling features in the Ising model can be incorporated into a Fisher-like scaling with $\Delta \mu=0$, which is obeyed rigorously over the explored temperature range below the critical temperature. Thus the observed clusters can be interpreted as a manifestation of the non-ideality of a vapor in equilibrium with a liquid. Finally, nuclear multifragmentation, which is seen to share all the scaling observed here, should be similarly interpreted as the clusterization of a nuclear vapor in equilibrium with its liquid.

\section{EXPERIMENTAL PROPERTIES OF THE SATURATED NUCLEAR VAPOR AND THE PHASE DIAGRAM}

Nuclear multifragmentation, the breakup of a nuclear system into several intermediate sized pieces, has been frequently discussed in terms of equilibrium statistical mechanics, and its possible association with a phase transition [42, 43]. However, to this point much uncertainty remains regarding its nature, in particular whether multifragmentation is a phase transition and if so, whether it is associated with the liquid to vapor phase transition. This paper will show that: 1) high quality experimental data contain the signature of a liquid to vapor phase transition through their strict adherence to Fisher's droplet model when modified to account for Coulomb energy; 2) the two-phase coexistence line is observed via the scaled fragment yields and extends over a large energy/temperature interval up to the critical point; 3) two critical exponents, as well as the critical temperature, the surface energy coefficient, enthalpy of evaporation and the critical compressibility factor can be directly extracted; and 4) the nuclear phase diagram can be constructed.

In past attempts to investigate the relationship between nuclear multifragmentation and a liquid to vapor phase transition, critical exponents have been determined [31, 42, 44, 45, 46], caloric curves have been examined [47], and the observation of negative heat capacities have been reported [27]. Other studies have shown two general, empirical properties of the fragment multiplicities called reducibility and thermal scaling [28, 29,30]. Reducibility, an indication of stochastic fragment production, refers to the observation that for each energy bin the fragment multiplicities are distributed according to a binomial or Poissonian law. As such, their multiplicity distributions can be reduced to a one-fragment production probability according to a binomial or Poissonian distribution. Thermal scaling, an indication of the thermal nature of fragment production, refers to the feature that the average fragment yield $\langle N\rangle$ behaves with temperature $T$ as a Boltzmann factor: $\langle N\rangle \propto \exp (-B / T)$.

The features of reducibility and thermal scaling are inherent to any statistical model. In particular they are present in Fisher's droplet model $[37,38,39,40]$ and will be observed in any system that it describes $[31,41,48]$. Fisher's model describes the aggregation of molecules into clusters in a vapor, thus accounting for its non-ideality. The abundance of a cluster of $\operatorname{size} A$ is given by:

$$
n_{A}=q_{0} A^{-\tau} \exp \left(\frac{A \Delta \mu}{T}-\frac{c_{0} \varepsilon A^{\sigma}}{T}\right),
$$

where $n_{A}=N_{A} / A_{0}$ is the number of droplets $N_{A}$ of mass $A$, normalized to the system size $A_{0} ; q_{0}$ is a normalization constant depending only on the value of $\tau$ [49]; $\tau$ is the topological critical exponent; $\Delta \mu=\mu-\mu_{l}$, and $\mu$ and $\mu_{l}$ are the actual and liquid chemical potentials respectively; $c_{0} \varepsilon A^{\sigma}$ is the surface free energy of a droplet of size $A ; c_{0}$ is the zero temperature surface energy coefficient; $\sigma$ is the critical exponent related to the ratio of the dimensionality of the surface to that of the volume; and $\varepsilon=\left(T_{c}-T\right) / T_{c}$ is the control parameter, a measure of the distance from the critical point, $T_{c}$. At coexistence $(\Delta \mu=0)$, Eq. (15) reduces to a Boltzmann factor with a "barrier" $B=c_{0} A^{\sigma}$ (the cost to produce the surface of a cluster of size $A$ ) and thermal scaling is observed.

Recently, multifragmentation data from the Indiana Silicon Sphere (ISiS) Collaboration was shown to exhibit both reducibility and thermal scaling [50,51], thus it may be interesting to determine if Fisher's model describes the ISiS data as well. In order to find if this is the case, the ISiS charge yields from AGS experiment E900a of $8 \mathrm{GeV} / \mathrm{c} \pi$ + Au fragmentation data (see Fig. 4a) were fit to the following modified form of Eq. (15) which incorporates, in an approximate manner, the Coulomb energy release when a particle moves from the liquid to the vapor:

$$
n_{A}=q_{0} A^{-\tau} \exp \left(\frac{A \Delta \mu+E_{C o u l}}{T}-\frac{c_{0} \varepsilon A^{\sigma}}{T}\right),
$$

where $E_{\text {Coul }}$ is given by:

$$
E_{\text {Coul }}=\frac{e^{2}}{4 \pi \varepsilon_{0}} \frac{\left(Z_{0}-Z\right) Z}{r_{0}\left(\left(A_{0}-A\right)^{1 / 3}+A^{1 / 3}\right)}\left(1-e^{-x \varepsilon}\right) .
$$



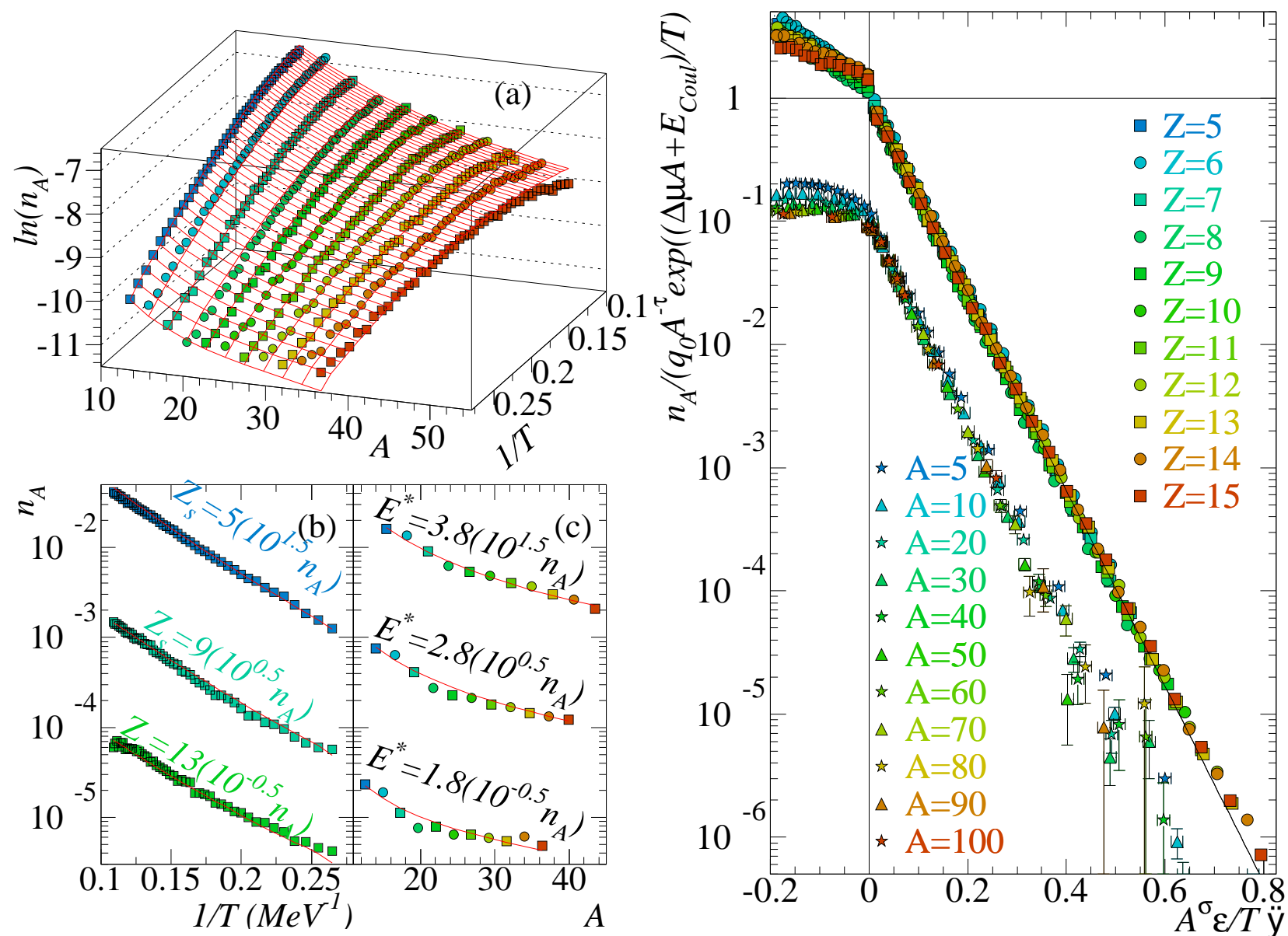

FIGURE 4. Left: (a) The ISiS fragment yield surface: natural log of the fragment yield vs. fragment mass and inverse temperature. (b) Arrhenius plots for representative charges. (c) Fragment mass yields for various values of $E^{*}$. Solid curves are from a fit to Fisher's model. Error bars are smaller than the size of the points. Right: The scaled yield distribution versus the scaled temperature for the ISiS data (upper) and $d=3$ Ising model calculation (lower). For the Ising model, the quantity $\left(n_{A} / q_{0} A^{-\tau}\right) / 10$ is plotted against the quantity $A^{\sigma} \varepsilon / 1.435 T$. Data for $T>T_{c}$ is scaled only as $n_{A} / q_{0} A^{-\tau}$.

Here $Z_{0}$ is the charge of the fragmenting system and $r_{0}=1.2 \mathrm{fm}$. This energy vanishes as $x \varepsilon$ at the critical point where no distinction exists between liquid and vapor. The mass of a fragment prior to secondary decay $A$ was estimated by multiplying the measured fragment charge $Z$ by an $A / Z$ ratio of 2 and by a factor of $\left(1+\left(E^{*} / B_{f}\right)\right)$ where $E^{*}$ is the reconstructed excitation energy of the event and $B_{f}$ is the binding energy of the fragment. The temperature $T$ was determined by assuming a degenerate Fermi gas, thus $T=\sqrt{E^{*} / a}$ and $a=A_{0} / \alpha ; \alpha=8\left(1+E^{*} / B_{0}\right)$ [52] with $B_{0}$ as the binding energy of the fragmenting system. This accommodates the empirically observed change in $\alpha$ with excitation energy [53].

Over 500 data points for $1.5 \leq E^{*} \leq 6.0 \mathrm{MeV} /$ nucleon and $5 \leq Z \leq 15$ were simultaneously fit to Eq. (16) with the parameters of the modified Fisher model $\left(\Delta \mu, x, \tau, \sigma, c_{0}\right.$ and $T_{c}$ ) allowed to vary to minimize chi-squared (see Fig. 4a). Fragments with $Z<5$ were not considered in the fit because: (1) Fisher's model expresses the mass/energy of a fragment in terms of bulk and surface energies and this approximation is known to fail for the lightest of nuclei where structure details (shell effects) dominate, and (2) for the lightest fragments equilibrium and non-equilibrium production cannot always be differentiated. Fragments with $Z>15$ were not elementally resolved [54], and were also excluded.

While analyses have been performed on other multifragmentation data in the past [55, 56, 57], those efforts dealt with inclusive data sets; data from every excitation energy were examined as a whole and the results were presented as a function of incident beam energy. The present work makes use of the high statistics, exclusive ISiS E900a data set and bins the events in terms of their reconstructed excitation energy [58]. In addition, explicit use of Fisher's 

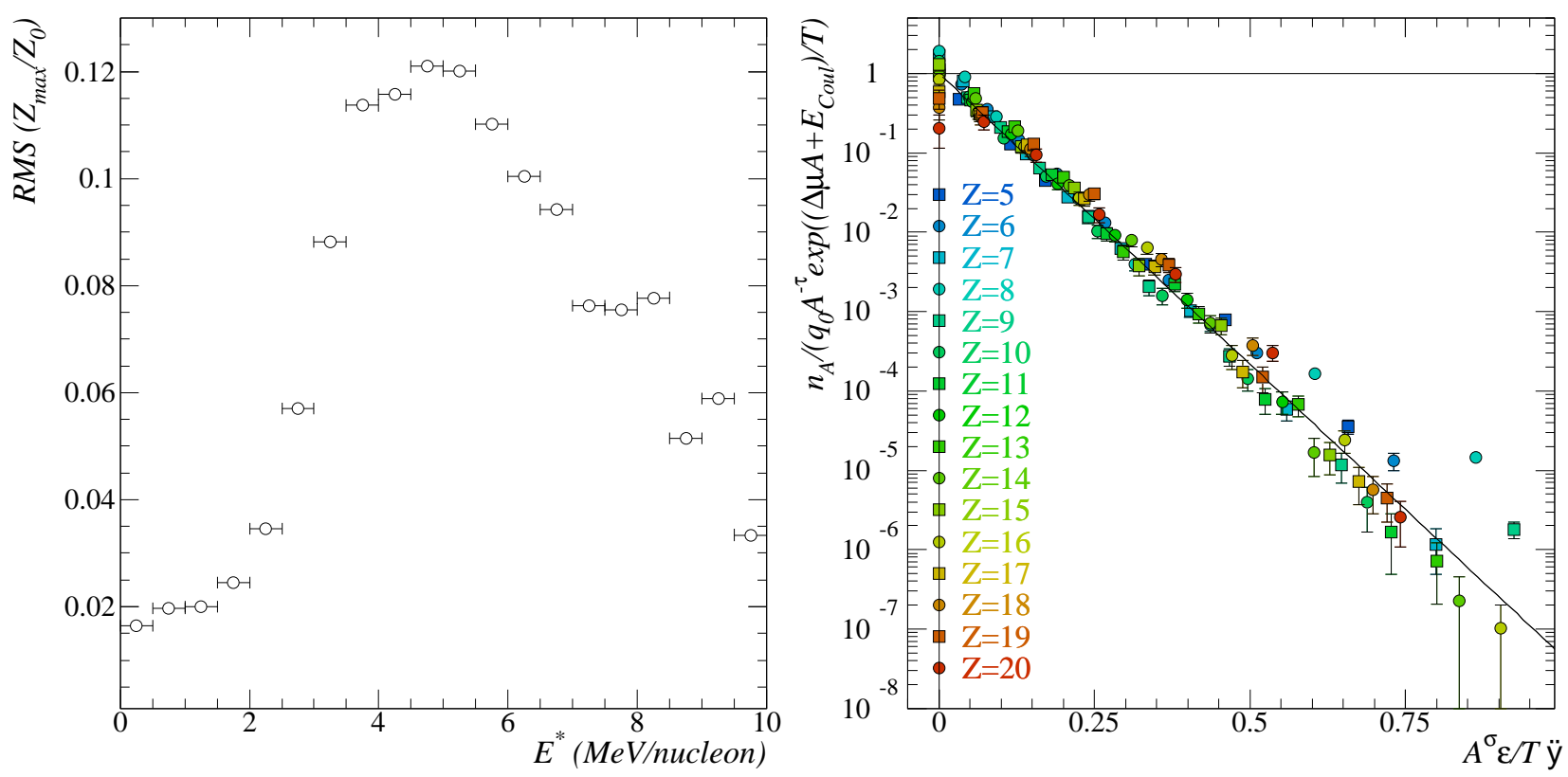

FIGURE 5. Left: RMS fluctuations of the charge of the largest fragment normalized to the charge of the fragmenting system plotted as a function of the excitation energy. Right: The scaled yield distribution versus the scaled temperature for the EOS Au+C data.

expressions for the bulk and surface energies and the inclusion of $E_{\text {Coul }}$ allows $\Delta \mu$ and $c_{0}$ to be determined directly from the data.

The behavior of the data for the $\left(n_{A}, A, T\right)$ surface is reproduced over a wide range in $E^{*}$ and $Z$ as shown in both Arrhenius plots (Fig. 4b) and fragment yield distributions (Fig. 4c). The results of scaling the data according to Eq. (16) are shown in Fig. 4. The fragment mass yield distribution is scaled by the Fisher's power law pre-factor, the bulk term and the Coulomb energy: $n_{A} / q_{0} A^{-\tau} \exp \left(\Delta \mu A+E_{\text {Coul }} / T\right)$. This quantity is plotted against the temperature scaled by Fisher's parameterization of the surface energy: $A^{\sigma} \varepsilon / T$. The scaled data collapse to a single line over six orders of magnitude, precisely the behavior predicted by Fisher's model. This line is the liquid-vapor coexistence line, as shown below, and provides direct evidence for the liquid to vapor phase transition in excited nuclei. It may be worth noticing that Fig. 4 represents the first extensive test ever for any physical system of Fisher's formula (Eq. (15)).

The value of $\tau=2.18 \pm 0.14$ is in the range predicted by Fisher's model and $\sigma=0.54 \pm 0.01$ is close to the value expected for a three dimensional system, $\sim 2 / 3$. The $\Delta \mu=0.06 \pm 0.03$, may indicate that the system is a slightly super-saturated vapor. The value of $x$ is $1.00 \pm 0.06$. The value of $c_{0}=18.3 \pm 0.5 \mathrm{MeV}$ is close to the value of the surface energy coefficient of the liquid-drop model: $16.8 \mathrm{MeV}$. The values of the critical exponents determined here are in agreement with those determined previously from other multifragmentation data $[45,46]$ and the value of the excitation energy at the critical point $E_{c}^{*}=3.8 \pm 0.3 \mathrm{MeV} /$ nucleon is in the neighborhood of the value observed in the EOS analysis $\left(E_{c}^{*} \approx 4.75 \mathrm{MeV} /\right.$ nucleon) $[31,46,59]$. The two experiments use a different method to distinguish particles resulting from the initial projectile-target collision from fragments formed afterwards; this difference leads to ${ }^{E O S} E^{*} \approx 1.2^{I S i S} E^{*}$ [58]. The value of ${ }^{E O S} E_{c}^{*}$ corresponds to the steepest decrease in the mass of the largest fragment and to the maximum value of its variance in the EOS data $[46,59]$. The extracted critical temperature $T_{c}=6.7 \pm 0.2$ $\mathrm{MeV}$ is comparable to theoretical estimates for small nuclear systems [60, 61, 62].

A previous analysis of the EOS $\mathrm{Au}+\mathrm{C}$ data relied on the assumptions that $\Delta \mu \approx 0$ and that the effects of the Coulomb energy were small $[31,46]$. The present, more general, analysis was performed on that data set. See Fig. 5. Here the the critical point was fixed by locating the maximum of the fluctuations of the charge of the largest fragment, $E_{c}^{*}=5.0 \pm 0.5 \mathrm{MeV} /$ nucleon. The parameters returned by a preliminary analysis of the EOS data agree well with those returned by the ISiS data when the difference in energy scales is taken into account: $T_{c}=8.3 \pm 0.6 \mathrm{MeV}$, $\Delta \mu=0.37 \pm 0.03 \mathrm{MeV} /$ nucleon, $c_{0}=16.9 \pm \mathrm{MeV}, \tau=2.37 \pm 0.06$ and $\sigma=0.58 \pm 0.02$.

Using the values of the parameters determined above for the ISiS experiment and Eq. (16), the coexistence curve observed in the scaled fragment yields in Fig. 4 can be cast into a more familiar form. Fisher's model assumes that 

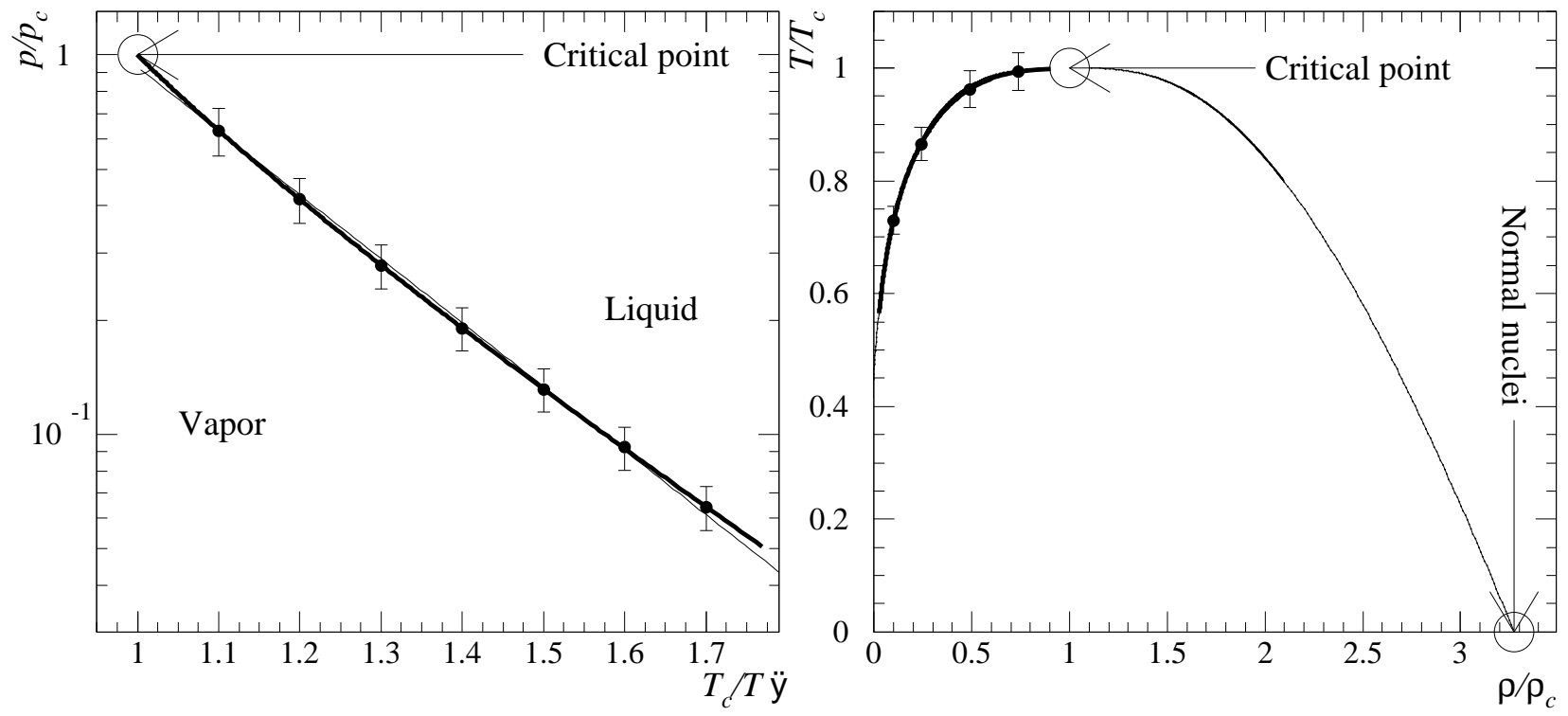

FIGURE 6. Left: The reduced pressure-temperature phase diagram: the thick line shows the calculated coexistence line, the points show selected calculated errors and the thin line shows a fit to the Clausius-Clapeyron equation. Right: The reduced densitytemperature phase diagram: the thick line shows the calculated low density branch of the coexistence curve, the points show selected calculated errors and the thin lines show a fit to and reflection of Guggenheim's equation.

the non-ideal vapor can be approximated by an ideal gas of clusters. Accordingly, the quantity $n_{A}$ is proportional to the partial pressure of a fragment of mass $A$ and the total pressure due to all of the fragments is the sum of their partial pressures: $p / T=\sum n_{A}$. In the actual experiment, this pressure is virtual, it is the pressure the vapor would have, to provide the back flow needed to keep the source at equilibrium. The reduced pressure is given by:

$$
\frac{p}{p_{c}}=\frac{T \sum n_{A}(T)}{T_{c} \sum n_{A}\left(T_{c}\right)} .
$$

The coexistence line for finite neutral nuclear matter is then obtained by using the $n_{A}\left(T, \Delta \mu=0, E_{\text {Coul }}=0\right)$ from Eq. (16) in Eq. (18). This is shown in Fig. 6. Recalling the Clausius-Clapeyron equation: $d p / d T=\Delta H / T \Delta V$ one obtains: $p / p_{c}=\exp \left(\Delta H / T_{c}\left(1-T_{c} / T\right)\right)$ which describes several fluids up to $T_{c}$ [63]. Fitting the coexistence line and using the above value of $T_{c}$ gives $\Delta H=26 \pm 1 \mathrm{MeV}$, the enthalpy of evaporation of a cluster from the liquid. This value, after a correction $p V=T$, gives a value for $\Delta E \approx 22 \mathrm{MeV}$. Since the gas described by Fisher's model is nonideal, the average cluster is greater in size than a monomer. The average size of a fragment in the region of the $p-T$ coexistence line obtained from Eq. (16) and the experimentally determined parameters is 1.5 . Thus the $\Delta E /$ nucleon becomes $\approx 15 \mathrm{MeV}$, remarkably close to the nuclear bulk energy coefficient.

The system's density can be found from $\rho=\sum A n_{A}$, and the reduced density from

$$
\frac{\rho}{\rho_{c}}=\frac{\sum A n_{A}(T)}{\sum A n_{A}\left(T_{c}\right)} .
$$

With $\Delta \mu$ and $E_{C o u l}$ set to 0 in Eq. (16), Eq. (19) yields the low density branch of the coexistence curve of finite neutral nuclear matter, shown in Fig. 6. Following Guggenheim it is possible to determine the high density branch as well: empirically, the $\rho / \rho_{c}-T / T_{c}$ coexistence curves of several fluids can be fit with the function [64]:

$$
\frac{\rho_{l, v}}{\rho_{c}}=1+b_{1}\left(1-\frac{T}{T_{c}}\right) \pm b_{2}\left(1-\frac{T}{T_{c}}\right)^{1 / 3}
$$

where the parameter $b_{2}$ is positive (negative) for the liquid $\rho_{l}$ (vapor $\rho_{v}$ ) branch. It was later recognized that the power of $1 / 3$ was the critical exponent $\beta$. Using Fisher's model, $\beta$ can be determined from $\tau$ and $\sigma: \beta=(\tau-2) / \sigma$ $[37,38,39,40]$. For this work $\beta=0.33 \pm 0.25$. Using this value of $\beta$ and fitting the coexistence curve from the ISiS 


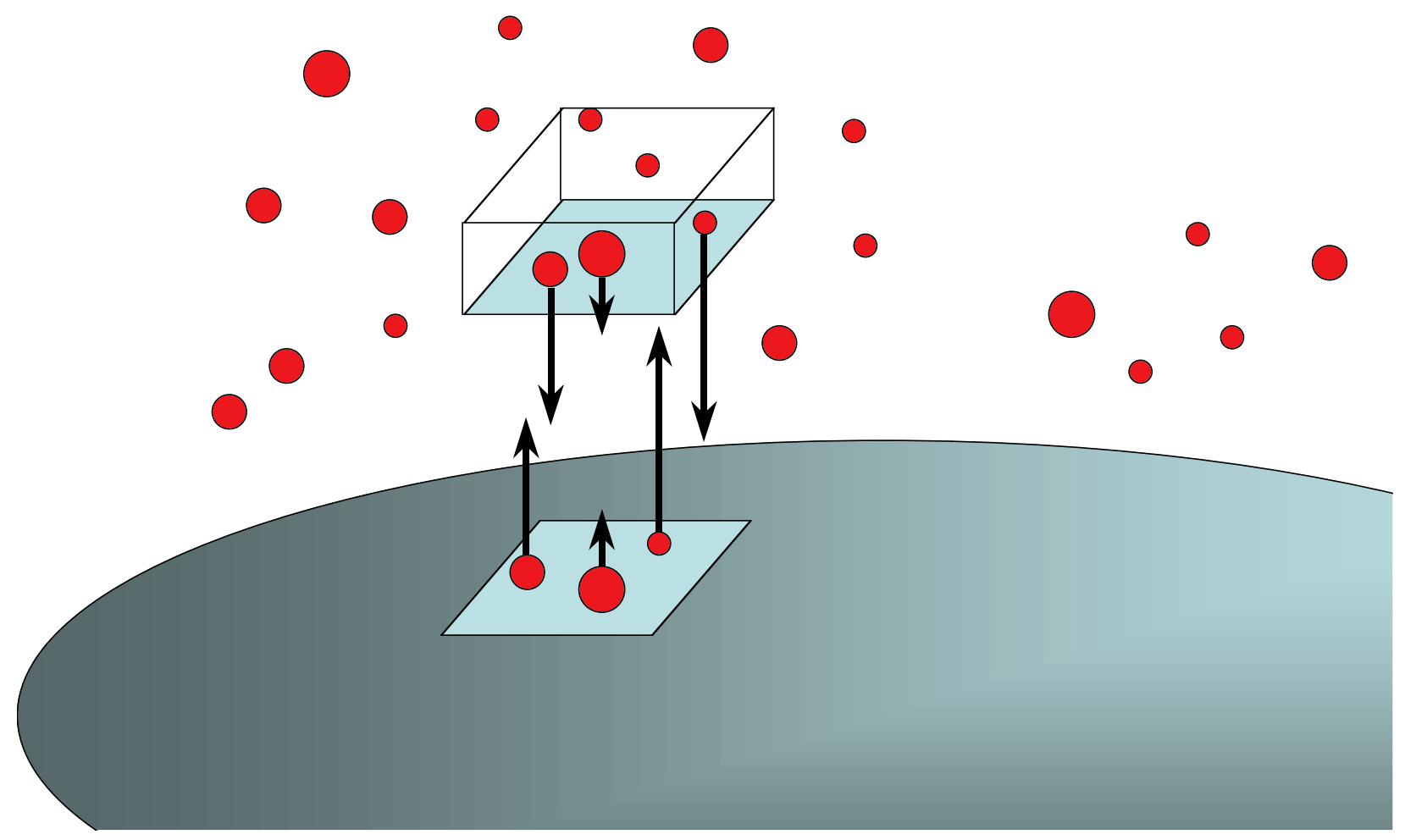

FIGURE 7. Emission of nuclear droplets from a compound nucleus.

E900a data with Eq. (20) one obtains an estimate of the full $\rho_{v}$ branch of the coexistence curve and changing the sign of $b_{2}$ gives the full $\rho_{l}$ branch of the coexistence curve of finite neutral nuclear matter. If normal nuclei exist at the $T=0$ point of the coexistence curve and the parameterization of the coexistence curve in Eq. (20) is used, then the critical density is found to be $\rho_{c} \sim 0.3 \rho_{0}$.

The critical compressibility factor $C_{c}^{F}=p_{c} / T_{c} \rho_{c}$ is found to be $0.25 \pm 0.06$, in agreement with the values for several fluids [65]. Using $T_{c}$ and $\rho_{c}$ from above in combination with $C_{c}^{F}$ gives a critical pressure of $p_{c} \sim 0.07 \mathrm{MeV} / \mathrm{fm}^{3}$.

\section{COMPOUND NUCLEUS DECAY AND THE LIQUID TO VAPOR PHASE TRANSITION}

The construction of a phase diagram, and in particular of a pressure-temperature diagram for a nuclear system leads us to the inevitable question: What is the meaning of pressure when the nuclear system is facing vacuum? This question has presented itself in many equivalent guises in the literature and in endless discussions. It amounts to asking: a) whether there is a gas phase in equilibrium with a liquid for the reactions in question; and b) whether this gas phase is thermodynamically characterizable.

The answer is no to a) and yes to b). And this is not contradictory. Consider the interface between a liquid and saturated vapor. From the liquid side we can specify with standard theories (e.g. compound nucleus decay rate, the equation for electrons emitted from a hot filament, etc.) the emission flux of particles from the surface. See Fig. 7. From the vapor side, we can write down the return flux into the liquid knowing the temperature, pressure/concentration and composition of the vapor. At equilibrium, by definition, the vapor to liquid flux matches physically and chemically the liquid to vapor flux. Thus, the saturated vapor acts, so to speak, as a mirror reflecting back elastically all the particles emitted by the liquid. This is the only role of the vapor.

If we remove the vapor, the liquid continues emitting particles as if the vapor were still present. Thus, the saturated vapor is completely characterized by the flux from the liquid side, even if the vapor itself is not physically there. So it is that we can unequivocally speak of the phase transition for a glass of water (or a nucleus) evaporating in a dry atmosphere or equivalently in vacuum. 


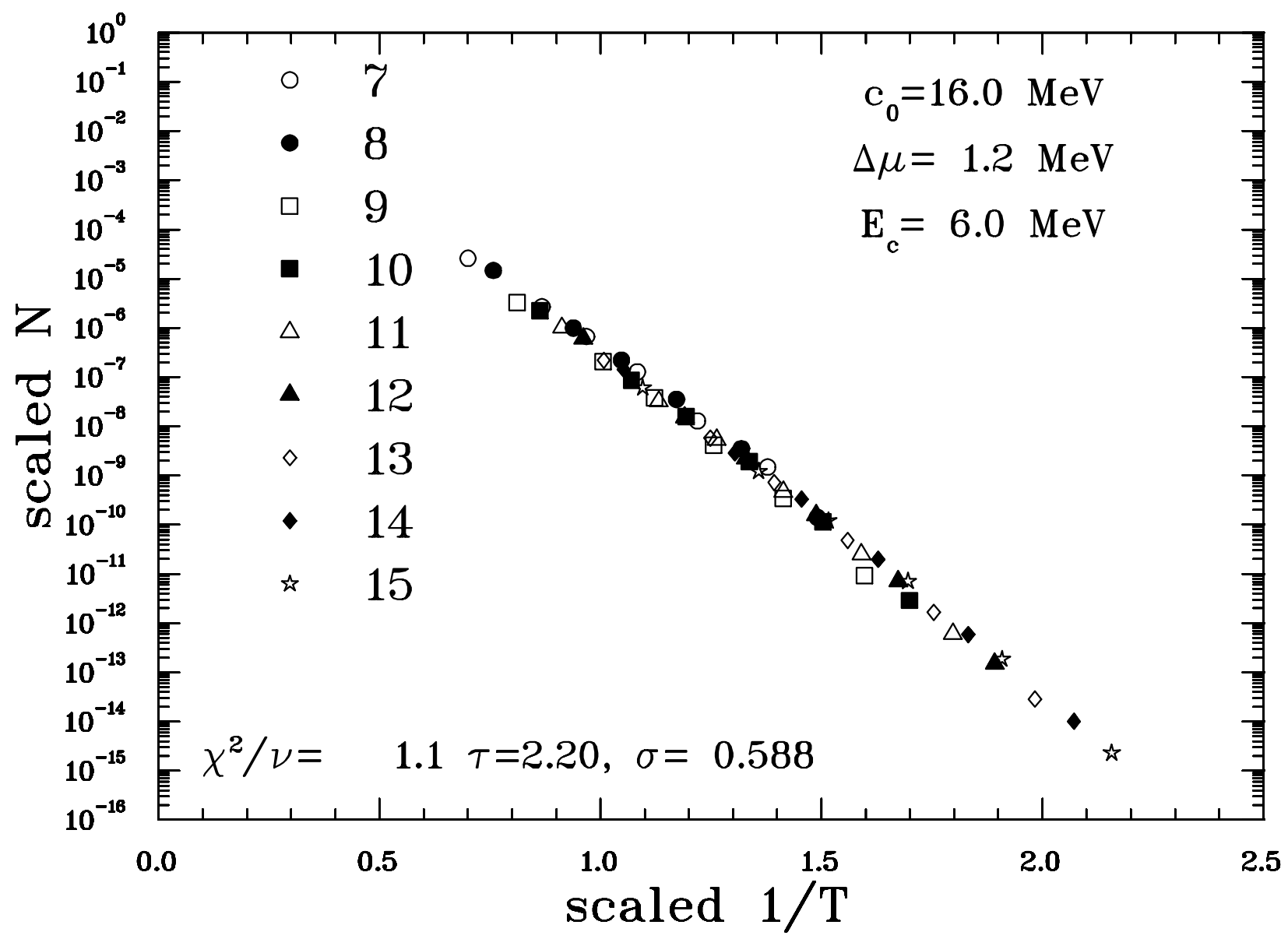

FIGURE 8. Preliminary results for the scaled yield distribution versus the scaled temperature for the $\mathrm{Ni}+\mathrm{C}$ low energy compound nucleus decay data.

In this light, compound nuclear decay becomes suddenly relevant to the liquid to vapor phase transition. In the past, we have studied the evaporation of complex fragments from well characterized compound nuclei [66]. It should be possible to cast these results in terms of Fisher's scaling. This is done in Fig. 8 for the reaction of $\mathrm{Ni}+\mathrm{C}$. As in the previous cases, the scaling is very good and the extracted parameters very close to those of the other systems. From this example we see in these low energy reactions a very interesting source for further characterization of the phase transition, in particular for anchoring the parameters of Fisher's model to the well established $T=0$ parameters of the liquid drop model.

In conclusion, the ISiS and EOS data, together with the low energy compound nucleus reaction contain the signature of a liquid to vapor phase transition via their strict adherence to Fisher's model. Through Fisher's scaling of the fragment yield distribution (Fig. 4), the two-phase coexistence line has been determined over a large energy/temperature interval extending up to the critical point. Fisher's formula (Eq. (15)) has been extensively tested and verified for the first time for any physical system. The critical exponents $\tau$ and $\sigma$ as well as the critical temperature $T_{c}$, the surface energy coefficient $c_{0}$, the enthalpy of evaporation $\Delta H$ and the critical compressibility factor $C_{c}^{F}$ have been extracted and found to agree with accepted values. Finally, $p_{c}$ and $\rho_{c}$ have also been determined, giving the first complete experimental determination of the critical point and the full phase diagram of finite neutral nuclear matter. 


\section{ACKNOWLEDGMENTS}

We thank Prof. C.M. Mader for her input and Ising model calculations. This work was supported by the US Department of Energy. We would also like to acknowledge the experimental and data reduction efforts of the ISiS and EOS collaborations, which made this analysis possible.

\section{REFERENCES}

1. D. Stauffer, Phys. Rev. Lett. 35, 394 (1975).

2. C. Domb and E. Stoll, J. Phys. A 10, 1141 (1977).

3. A. Coniglio and W. Klein, J. Phys. A 13, 2775 (1980).

4. J. L. Cambier and M. Nauenberg, Phys. Rev. B 34, 8071 (1986).

5. J. Kertész, Physica A 161, 58 (1989).

6. J. S. Wang, Physica A 161, 249 (1989).

7. J. S. Wang and R. H. Swendsen, Physica A 167, 565 (1990).

8. D. De Meo, D. W. Heermann and K. Binder, J. Stat. Phys. 60, 585 (1990).

9. A. M. Ferrenberg and D. P. Landau, Phys. Rev. B 44, 5081 (1991).

10. J. J. Alonso, A. I. López-Lacomba and J. Marro, Phys. Rev. E 52, 6006 (1995).

11. J. Kertesz, A. Coniglio, and D., Stauffer, Ann. Israel Phys. Soc. p.121 (1997).

12. A. Coniglio, Nucl. Phys. A 681, 451c (2001).

13. T. D. Lee and C. N. Yang, Phys. Rev. 87, 404 (1952).

14. T. D. Lee and C. N. Yang, Phys. Rev. 87, 410 (1952).

15. S. K. Samaddar and J. Richert, Phys. Lett. B 218381 (1989).

16. S. K. Samaddar and J. Richert, Z. Phys. A 332443 (1989).

17. J. Pan and S. Das Gupta, Phys. Lett. B 34429 (1995).

18. J. Pan and S. Das Gupta, Phys. Rev. C 511384 (1995).

19. S. Das Gupta and J. Pan, Phys. Rev. C 531319 (1996).

20. X. Campi and H. Krivine, Nucl. Phys. A 62046 (1997).

21. X. Campi, H. Krivine and A. Puente, Physica A 262328 (1999).

22. F. Gulminelli and Ph. Chomaz, Phys. Rev. Lett. 821402 (1999).

23. Ph. Chomaz and F. Gulminelli, Phys. Lett. B 447221 (1999).

24. V. Duflot, Ph. Chomaz and F. Gulminelli, Phys. Lett. B 476279 (2000).

25. Ph. Chomaz, et al., Phys. Rev. Lett. 85, 3587 (2000).

26. D. H. E. Gross, Phys. Rep. 279, 119 (1997).

27. M. D'Agostino et al., Phys. Lett. B, 473, 219 (2000).

28. L. G. Moretto, et al., Phys. Rep. 287, 249 (1997).

29. L. Beaulieu et al., Phys. Rev. Lett. 81, 770 (1998).

30. L. G. Moretto et al., Phys. Rev. C 60, 031601 (1999).

31. J. B. Elliott et al., Phys. Rev. Lett 85, 1194 (2000).

32. D. Stauffer, Phys. Rep 54, 2 (1979).

33. D. Stauffer and A. Aharony, "Introduction to Percolation Theory", 2nd ed. (Taylor and Francis, London, 1992).

34. W. Bauer et al., Phys. Lett. B 150, 53 (1985).

35. W. Bauer et al., Prog. Part. Nucl. Phys. 99, 42 (1999).

36. P. D. Gujrati, Phys. Rev. E 51, 957 (1995).

37. M. E. Fisher, Physics 3, 255 (1967).

38. M. E. Fisher, Rep. Prog. Phys. 30, 615 (1969).

39. C. S. Kiang and D. Stauffer, Z. Physik 235, 130 (1970).

40. D. Stauffer and C. S. Kiang, Advances in Colloid and Interface Science 7, 103 (1977).

41. J. B. Elliott et al., to be published in Phys. Rev. Lett (2001).

42. J.E. Finn et al., Phys. Rev. Lett. 49, 1321 (1982).

43. P.J. Siemens, Nature 305, 410 (1983).

44. M.L. Gilkes et al., Phys. Rev. Lett. 73, 1590 (1994).

45. M. D’Agostino et al., Nucl. Phys. A 650, 328 (1999).

46. J.B. Elliott et al., Phys. Rev. C 62, 064603 (2000).

47. J. Pochodzalla et al., Phys. Rev. Lett 75, 1040 (1995).

48. C.M. Mader et al, LBNL-47575, nucl-th/0103030 (2001).

49. H. Nakanishi et al., Phys. Rev. B 22, 2466 (1980).

50. L. Beaulieu et al., Phys. Rev. Lett. 84, 5971 (2000).

51. L. Beaulieu et al., Phys. Rev. C 63, 031302 (2001).

52. A.H. Raduta et al., Phys. Rev. C 55, 1344 (1997).

53. K. Hagel et al., Nucl. Phys. A486, 429 (1988). 
54. K. Kwiatkowski et al., NIM A360, 571 (1995).

55. A.S. Hirsch et al., Phys. Rev. C 29, 508 (1984).

56. A.L. Goodman et al., Phys. Rev. C 30, 851 (1984).

57. M. Mahi et al., Phys. Rev. Lett. 60, 1936 (1988).

58. T. Lefort et al., Phys. Rev. Lett 83, 4033 (1999).

59. J.A. Hauger et al., Phys. Rev. C 57, 764 (1998)

60. H.R. Jaqaman et al., Phys. Rev. C 29, 2067 (1984).

61. P. Bonche et al., Nucl. Phys. A 436, 265 (1985).

62. J. N. De et al., Phys. Rev. C 59, R1 (1999).

63. E.A. Guggenheim, "Thermodynamics", 4th ed. (North-Holland, 1993).

64. E.A. Guggenheim, J. Chem. Phys., 13, 253 (1945).

65. C.S. Kiang, Phys. Rev. Lett. 24, 47 (1970).

66. T. S. Fan, et ap., Nucl. Phys. A 679, 121 (2000). 